\title{
La formation d'une fosse d'érosion dans un bassin de dissipation : une conséquence de la cavitation ?
}

\section{Formation of an erosion pit in a stilling basin : a consequence of cavitation?}

\author{
E. Frossard
}

Coyne et Bellier, Paris

\begin{abstract}
Dans un bassin recevant un jet en chute libre, se forme parfois une fosse d'érosion. L'article présente un modèle physique simple semi-quantitatif des effets de pression au fond du bassin, qui résultent de trois composantes: la pression statique (loi de débit de l'exutoire), la surpression dynamique moyenne (impact d'un jet stationnaire), la fluctuation autour de la valeur moyenne (turbulence).

A partir de caractéristiques des pressions moyennes et fluctuantes tirées de la littérature, le modèle donne analytiquement la profondeur minimale annulant le risque de cavitation sur le fond. Cette profondeur correspond, formellement et en ordre de grandeur, à la profondeur ultime d'érosion donnée par l'expression empirique classique de Véronèse.

Le moteur physique de ce mécanisme étant l'effet des forces vives, le rôle de la dissipation d'énergie est discuté.
\end{abstract}

\section{Notations}

A Constante adimensionnelle $A \cong 7,43$

$B \quad$ Epaisseur du jet à l'impact

C Constante (notation générique)

d Profondeur

F Fréquence au dépassement

$g \quad$ Intensité de la pesanteur

$\mathrm{H}, \mathrm{Hc} \quad$ Charges hydrauliques

$h_{s} \quad$ Charge d'eau correspondant à la pression de vapeur saturante

Constante
An erosion pit may form in a basin into which freely falls a hydraulic jump. This paper describes a simple, semiquantitative physical model of the pressure effects at the bottom of the basin, resulting from three components: static pressure (tailwater-rating curve), average dynamic overpressure (impact of a stationary jump), and fluctuation around the mean value (turbulence).

From the characteristics of the mean and fluctuating pressures taken from the literature, the model analytically gives the minimum depth eliminating the risk of cavitation on the bottom. Formally, and as an order of magnitude, this depth corresponds to the ultimate erosion depth given by Veronese's conventional empirical expression.

As the physical motor of this mechanism is the effect of momentum, the role of energy dissipation is discussed. 
Figure $1-$ La fosse d'érosion de Kariba en 1981.

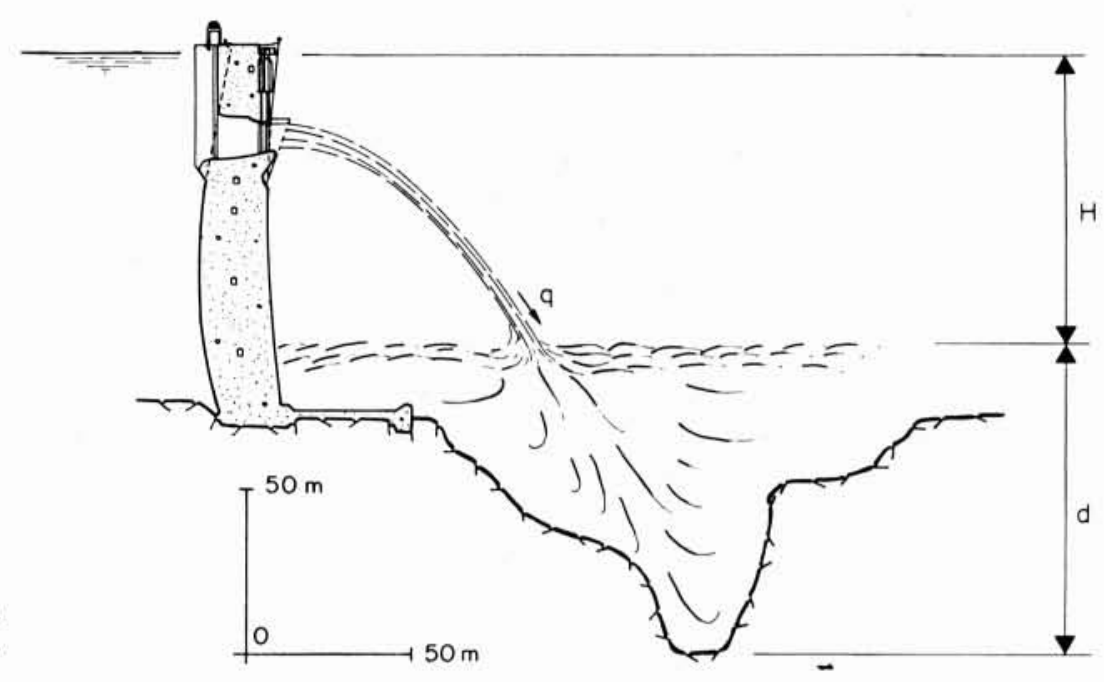

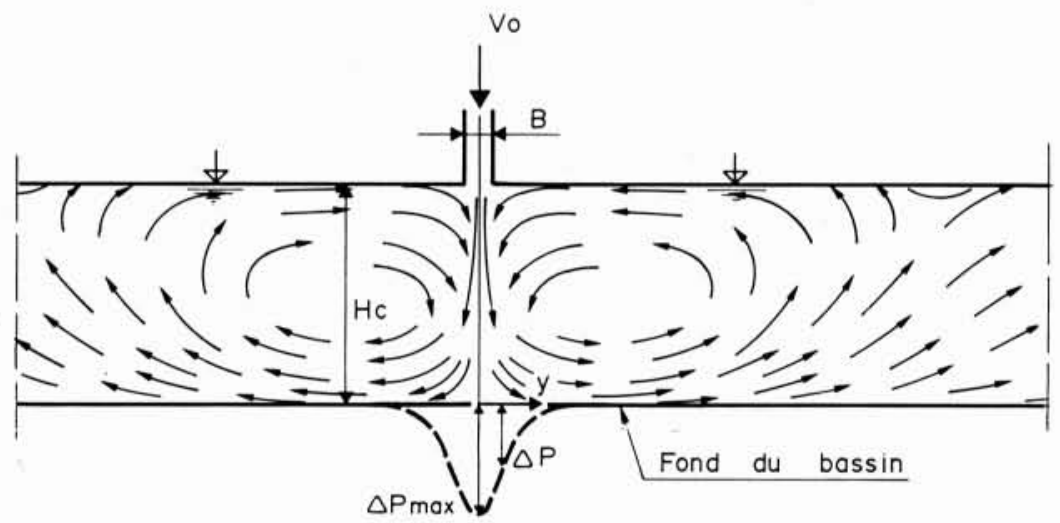

Figure 2 - Distribution de la surpression sur le fond d'un bassin soumis à l'impact d'un jet plan stationnaire.

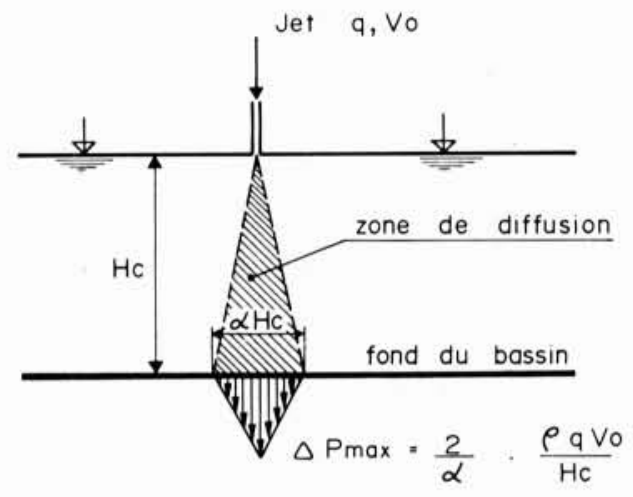

Figure 3 - Schématisation du modèle de Cola et $\mathrm{du}$ diagramme des surpressions dynamiques moyennes sur le fond $\alpha=4 / A \cong 0,54$.

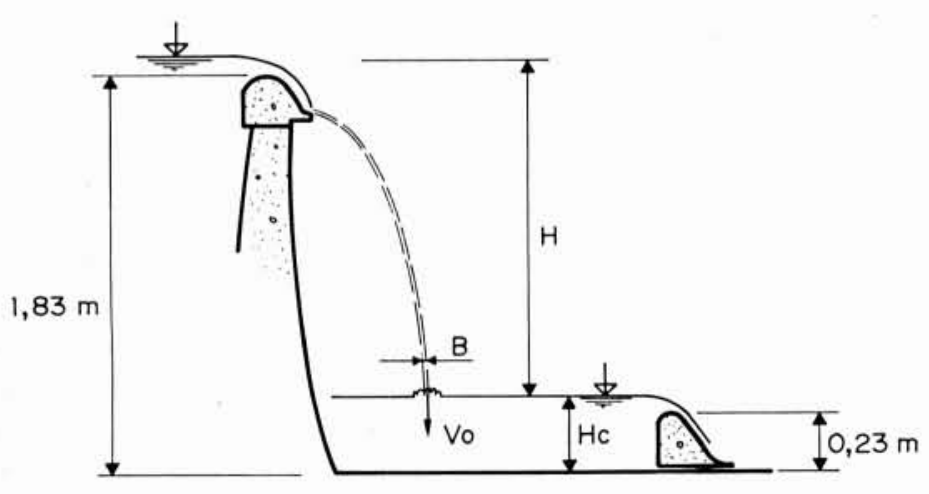

Figure 4 - Schéma du modèle de Franzetti.

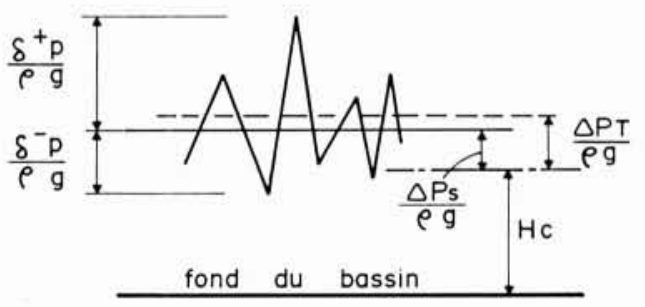

Figure 5 - Diagramme type des fluctuations de pression.

Hc Cote piézométrique théorique par rapport au fond, calculée par la loi de débit de l'exutoire.

$\Delta P_{S}$ Surpression dynamique moyenne expérimentale.

$\Delta P_{T}$ Surpression dynamique moyenne théorique.

$\delta^{+} p$ Pic de surpression par rapport à la moyenne expérimentale.

$\delta^{-} p$ Pic de dépression par rapport à la moyenne expérimentale. 


\section{Introduction}

- Le fonctionnement d'un bassin de dissipation recevant un jet en chute libre, comme il en existe à l'aval des évacuateurs de crue de nombreux barrages, peut conduire à l'excavation progressive d'une fosse d'érosion (fig. 1).

La profondeur ultime d'une telle fosse est souvent évaluée, au stade préliminaire des projets, par la formule de A. VERONESE (1937) [1] qui correspond au cas d'un jet en nappe comme il s'en produit sous un déversoir long :

$$
d=1,9 q^{0.54} H^{0.225}
$$

oủ $q$ est le débit unitaire à l'impact.

- E. ScImemi (1947) [2] a montré par des expériences sur modèle que la profondeur ultime donnée par la formule de VERONESE est atteinte lorsque tend vers zéro la granularité moyenne (le " $\left.D_{50} »\right)$ des éléments formant le matériau du fond, sans cohésion.

D'autres auteurs [3] concluent que cette profondeur est, à long terme, indépendante de la taille des éléments formant le fond, du fait de l'action abrasive des éléments entrainés dans les tourbillons.

- Divers auteurs ont proposé d'autres expressions pour cette profondeur, dont notamment MARTINS (1975) [4], Machado (1982) [5], Chandra et Agarwal (1982) [6], MASON (1984) [7], et LenCASTRE (1985) [8].

La dispersion des diagrammes de comparaison entre les valeurs calculées et les résultats observés ne semble pas toujours en rapport avec la complexité des expressions proposées, qui tentent cependant de tenir compte de la nature du fond. Ces diverses formules, très dissemblables dans leur apparence, présentent toutefois quelques traits communs : les variables essentielles semblent être le débit unitaire à l'impact $q$ et la hauteur de chute $H$; le poids relatif du débit unitaire étant plus fort que celui de la hauteur de chute, d'un facteur 2 voire davantage. La variété de ces formules souligne d'une part la variété des expérimentations, et d'autre part celle des méthodes employées pour rassembler les résultats en une expression analytique. L'auteur, qui n'est pas spécialiste, ne s'attachera pas à les détailler davantage.

- L'objet du présent article est de présenter un schéma physique simple fondé sur une analyse des fluctuations de pression et sous-jacent à la première de ces expressions : le résultat empirique de VERONESE. Comme il a été suggéré plus haut, la bibliographique sur le sujet parait avare en modèles physiques simples semi-quantitatifs, c'est pourquoi l'auteur propose ce schéma qui, de par sa simplicité, ne saurait prétendre à une précision mécanique. Les idées présentées ici sont fondées sur les travaux publiés dans l'Energia Elettrica par R. Cola (1966) [9] et S. FRANZETTI (1980) [10], elles ont pris forme durant la conception d'ouvrages d'évacuation de crues en 1982 et sont originales, à la connaissance de l'auteur.

\section{Effets de pression dus à l'impact du jet}

- Le fonctionnement d'un bassin de dissipation s'accompagne d'un pilonnage des parois par des fluctuations de pression associées à une forte turbulence.

Les effets de pression dynamique sur le fond, dûs à la diffusion du jet dans la masse d'eau du bassin, ont été détaillés par R. CoLA [9] pour la valeur moyenne, et S. FRANZETTI [10] pour les fluctuations instantanées.

\section{Pression dynamique moyenne}

- Les effets de diffusion de jets submergés ont été étudiés par divers auteurs, dont Albertson, Rouse et al [11].

COLA a repris ces résultats pour étudier la surpression dynamique moyenne entretenue par la diffusion d'un jet plan stationnaire tombant verticalement dans un bassin à fond plat (fig. 2).

Le résultat essentiel, obtenu par l'analyse théorique de l'écoulement et vérifié par les expériences de ces auteurs, est l'expression de la surpression moyenne entretenue sur le fond:

$$
\Delta P=\Delta P_{\operatorname{Max}} \cdot e^{-\kappa\left(y / H()^{2}\right.}
$$

avec

$$
\Delta P_{\text {Max }}=A \cdot \rho V_{0}^{2} B / 2 H c
$$

où $A \cong 7,43$ et $K=\pi A^{2} / 4$ sont des constantes

Ces expressions sont relatives au cas d'un bassin dont la profondeur $H c$ est supérieure à une "profondeur de pénétration du jet " qui est de l'ordre de $A . B \cong 7,4$. B.

Les bassins de dissipation auxquels on s'intéresse dans cet article vérifient largement cette condition.

- Il est utile de noter que l'expression de $\Delta P_{\text {Max }}$ (équ. (3)) contient deux aspects physiques:

- la surpression dynamique moyenne résulte des forces qui naissent de la déflexion du jet, par un effet de quantité de mouvement qui se traduit, au numérateur de (3), par le produit $\rho V_{0}^{2} B=\rho q V_{0}$, quantité de mouvement unitaire à l'impact;

- l'effet de diffusion des vitesses moyennes, qui se trouve linéaire avec la profondeur, tend à étaler la surface où s'exerce cette surpression, et se traduit par le dénominateur $H c$.

Ces remarques conduisent au schéma de la figure 3, qui respecte la valeur maximale de COLA et l'équilibre des forces, et qui trouvera son utilité plus loin.

\section{Fluctuations de pression}

- S. Franzetti a réalisé une série de mesure de pression sur un modèle constitué par (fig. 4):

- un barrage déversant, de hauteur totale $1,83 \mathrm{~m}$,

- un bassin d'amortissement, de longueur 1,60 m environ,

- un contre-barrage de hauteur $0,23 \mathrm{~m}$.

Pour diverses valeurs de débit déversé, cet auteur a mesuré la pression sur le fond du bassin au moyen d'une série de capteurs piézorésitifs dont les signaux étaient enregistrés.

- Afin de traiter les mesures, Franzetti a distingué les grandeurs suivantes (fig. 5).

Les résultats essentiels publiés par cet auteur sont que :

- la surpression dynamique moyenne expérimentale $\Delta P_{S}$ coïncide approximativement avec la valeur théorique $\Delta P_{T}$ donnée par les formules de COLA, pourvu que le débit soit suffisamment élevé, 
- la distribution des pics de surpression ou de dépression est telle que, pour une fréquence au dépassement donnée $F$, la valeur expérimentale de la fluctuation correspondante $\delta p_{F}$ serait proportionnelle à la moyenne $\Delta P_{S}$, le coefficient de proportionnalité ne dépendant que de la fréquence $F$ et du signe des fluctuations considérées :

$$
\begin{aligned}
& \delta^{+} p_{F}(F, Q) \cong \lambda^{+}(F) \cdot \Delta P_{S}(Q) \\
& \delta^{-} p_{F}(F, Q) \cong \lambda^{-}(F) \cdot \Delta P_{S}(Q)
\end{aligned}
$$

- les fonctions $\lambda^{+}(F)$ et $\lambda^{-}(F)$ tendraient vers une limite finie $\lambda^{+}(0), \lambda^{-}(0)$, quand la fréquence $F$ tend vers zéro. Ceci revient à dire que les fluctuations les plus exceptionnelles de la pression locale instantanée, qui représente physiquement une énergie par unité de volume, resteraient bornées.

\section{Le schéma proposé}

- On peut penser que l'effet, sur la tenue du fond, du pilonnage par les pulsations de pression, dépend non seulement de leur valeur absolue, mais aussi de leur valeur relative par rapport à la pression moyenne, résultante des deux composantes $H c$ et $\Delta P_{S}$.

Un schéma simplifié des relations entre les pulsations de pression et $\Delta P_{S}$ ayant pu être tiré des résultats de FRANZETTI, il reste donc à examiner les relations entre les pulsations $\delta p$ et la pression statique $H c$.

Dans un premier temps, on s'attachera pour cela à la valeur relative des fluctuations maximales par rapport à la charge statique, et, dans un deuxième temps, à la profondeur jusqu'à laquelle les fluctuations en dépression sont susceptibles de provoquer la cavitation.

- D'après ce qui précède :

- les surpressions ou dépressions maximales sur le fond, en moyenne et en fluctuation, se produisent directement sous l'impact du jet dans le bassin,

- la valeur de ces fluctuations maximales vaudrait :

$\delta^{+}{ }_{\operatorname{Max}} p=\lambda^{+}(0) \cdot \Delta P_{T}$

$\delta^{-}{ }_{\text {Max }} p=\lambda^{-}(0) \cdot \Delta P_{T}$

- $\Delta P_{T}$ est donnée par la formule de Cola, équ. (3). Il est utile de remarquer que dans cette expression, le produit $V_{0} B$ représente le débit du jet par unité de longueur de la nappe déversante à l'impact, c'est-à-dire le débit unitaire $q$ à l'impact. Par ailleurs, la vitesse $v_{0}$ est liée à la hauteur de chute $H$ par

$$
\mathrm{V}_{0} \sim \sqrt{2 g H}
$$

Enfin, la cote piézométrique de la surface du bassin par rapport au fond $H c$, est égale à la profondeur $d$.

La formule de ColA s'écrit donc:

$$
\Delta P_{T}=A \cdot \rho \cdot \frac{\mathrm{q} \sqrt{2 g H}}{2 d}
$$

soit encore :

$$
\Delta P_{T} \cong 5,25 \cdot \rho \cdot \frac{\mathrm{q} \sqrt{g H}}{d}
$$

- Si l'on cherche donc quelle profondeur donner au bassin pour que l'amplitude de la fluctuation maximale en surpression - ou en dépression - n'excède pas la proportion $k$ de la charge statique $d$, on obtient :

- en surpression

$$
\frac{1}{\rho g} \cdot \delta_{\operatorname{Max}}^{+} p \leqslant \underline{k} \cdot d
$$

soit en tenant compte de (4) et (5),

$$
\lambda^{+}(0) \cdot \frac{5,25}{\sqrt{g}} \cdot \frac{q H}{d} \leqslant \underline{k} d
$$

qui s'écrit encore

$$
\underline{d} \geqslant C \cdot q^{1 / 2} \cdot H^{1 / 4}
$$

avec

$$
C=\sqrt{\frac{5,25 \lambda^{+}(0)}{k \sqrt{g}}}
$$

- en dépression, le raisonnement précédent reste valide, à condition de remplacer $\delta^{+}{ }_{\operatorname{Max}} p$ par $\delta^{-}{ }_{\operatorname{Max}} p$ et $\lambda^{+}(0)$ par $\lambda^{-}(0)$.

- La profondeur limite de cavitation est celle pour laquelle s'annule la résultante de :

- la charge hydrostatique $d$,

- la charge dynamique moyenne $\frac{1}{\rho g} \cdot \Delta P_{T}$

- la fluctuation maximale en dépression

$$
-\frac{1}{\rho g} \cdot \delta_{\operatorname{Max}} p
$$

soit :

$$
d+\frac{1}{\rho g} \cdot \Delta P_{T}-\frac{1}{\rho g} \delta_{\overline{M a x}}^{-} p=0
$$

En tenant compte de (4) et (5), cette équation (8) conduit encore à l'équation (6) :

$$
\underline{d}=C \cdot q^{1 / 2} \cdot H^{1 / 4}
$$

avec ici

$$
C=\sqrt{\frac{\left(\lambda^{-}(0)-1\right) \cdot 5,25}{\sqrt{g}}}
$$

Il ressort des résultats de Franzetti que $\lambda^{-}(0)>2,2$. En reportant cette valeur dans (9), on obtient donc comme profondeur limite de cavitation, dans un bassin d'amortissement à fond plat :

$$
\underline{d>1,4 \cdot q^{1 / 2} \cdot H^{1 / 4}}
$$

La cavitation étant l'apparition de bulles de vapeur, on pourrait tenir compte de l'effet de la pression de vapeur saturante $P s$ en remplaçant le second membre de (8) par $h_{s}=P s / \rho g$. On trouve alors comme solution $d=d_{0}\left(1+3 / 2 \cdot U-1 / 8 \cdot U^{2}+\ldots\right)$ où $d_{0}$ est la solution donnée par (6) et (9), et $U=1 / 2 \cdot h_{s} / d_{0}$ est un terme en général petit devant 1 . 
Déversement au barrage de l'Aigle sur la Dordogne (photo d'archives $C . \& B$.)

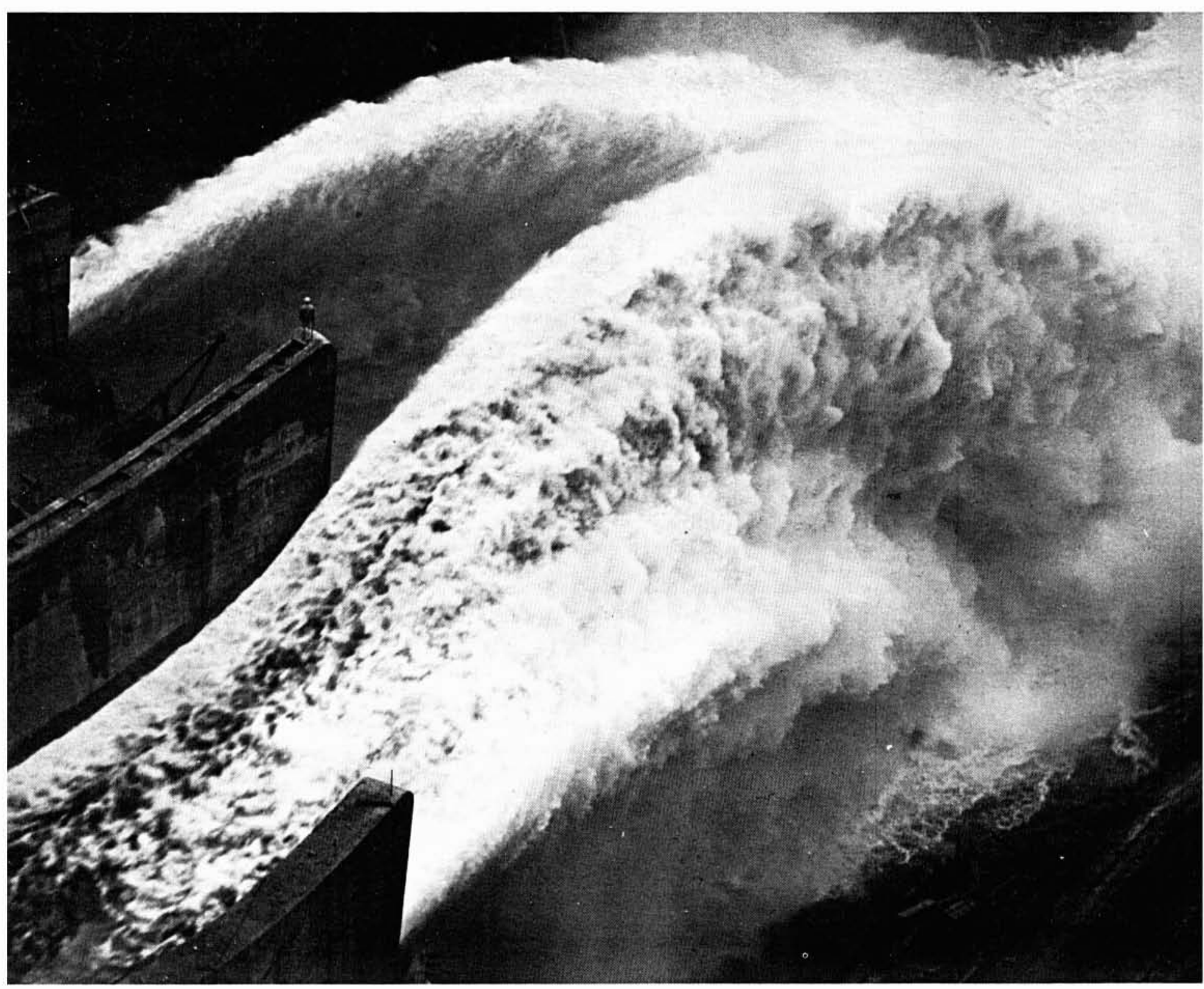


Le rapprochement de cette profondeur limite de cavitation

$$
\underline{d}>1,4 q^{0.5} \cdot H^{0.25}
$$

avec la profondeur ultime de la fosse d'érosion donnée par la formule de VERONESE:

$$
\underline{d}=1,9 q^{0.54} \cdot H^{0.225}
$$

désigne donc clairement le rôle essentiel joué par les fluctuations de pression en général et la cavitation en particulier.

Sur le plan numérique, cette profondeur de cavitation vaut entre $70 \%$ et $80 \%$ de la profondeur de VERONESE tant que $2 \mathrm{~m}^{3} / \mathrm{s} . \mathrm{m}<q<25 \mathrm{~m}^{3} / \mathrm{s} . \mathrm{m}$ et $20 \mathrm{~m}<H<100 \mathrm{~m}$.

- On peut chercher quelle est l'amplitude des fluctuations en surpression qui subsistent à la profondeur limite de cavitation.

A partir des relations (6), (7) et (9), on obtient directement :

$$
\underline{k}=\frac{\lambda^{+}(0)}{\lambda^{-}(0)-1}
$$

Les résultats de FranzetTI indiquent $\lambda^{+}(0)>7$ d'où $k \cong 5,8$.

On constate donc que, au fond d'un bassin d'amortissement à fond plat fonctionnant à la profondeur limite de cavitation, subsistent des fluctuations maximales en surpression qui seraient encore de l'ordre de 5 à 6 fois la charge statique.

Si la profondeur de fonctionnement du même bassin est celle de la formule de VERONESE, on trouve par le même raisonnement que les fluctuations maximales en surpression sur le fond seraient encore de l'ordre de 3 fois la charge statique, tandis que les fluctuations maximales en dépression y sont encore de l'ordre de $100 \%$ de la charge statique.

- On peut remarquer que, pour un bassin donné, la valeur limite observée par $\lambda^{-}(0)$ dépend certainement de la finesse de résolution dans le temps du système de mesure et d'enregistrement. Si donc il existe bien une valeur limite réelle $\lambda_{r}^{-}(0)$, de par sa définition, elle est supérieure aux valeurs observées, et la profondeur de non cavitation réelle est plus grande que la valeur calculée par la formule (10).

De plus, dans le cas d'une fosse étroite avec des parois raides et irrégulières, comme il peut s'en former dans un lit rocheux, (fig. 1), les lignes de courant sont déviées d'un angle plus proche de $180^{\circ}$ que de l'angle droit qui correspond au cas du bassin à fond plat, (fig. 2), et le $\Delta P_{\text {Max }}$ de la formule de COLA, qui traduit en pression l'effet de cette déviation, est à multiplier par un coefficient amplificateur pouvant aller jusqu'à 2 .

Enfin, dans une fosse en formation, l'essentiel de la diffusion des vitesses se produisant dans un domaine moins ouvert et moins régulier que dans un bassin à fond plat, le confinement de la turbulence y conduit peut être à des fluctuations plus fortes.

- Ces remarques suggèrent que, dans le cas de la géométrie d'une fosse, la profondeur limite de cavitation est vraisemblablement plus grande que la valeur donnée par la formule (10).

Cette conclusion rejoint celle de Machado [5], qui est fondée sur les essais de modèle hydraulique pour la fosse d'érosion du barrage voûte du Xingo, en projet au Brésil, qui devra évacuer un débit maximal de $33000 \mathrm{~m}^{3} / \mathrm{s}$ dans le canyon du Rio Sao Francisco, large de $100 \mathrm{~m}$ environ, avec une hauteur de chute $\mathrm{H}$ de $84 \mathrm{~m}$.

\section{Discussion}

- L'interprétation du mécanisme physique de formation des fosses d'érosion qui est proposée dans cet article résulte, au fond, du rapprochement entre les formules de Veronese et de Cola.

Il a été souligné plus haut que celle-ci décrivait l'effet de quantité de mouvement avec une diffusion linéaire en profondeur.

Le moteur de la formation des fosses serait donc cet effet de quantité de mouvement.

- Il paraît surprenant que n’apparaissent guère la notion de dissipation d'énergie, qui est pourtant la fonction même de ces bassins.

Sans entrer dans le détail de la dissipation associée aux structures turbulentes, dont un exposé de synthèse a été publié par Binder et al. (1980) [12], on peut faire les remarques suivantes:

- en admettant que l'essentiel de la dissipation a lieu dans la zone de diffusion du jet, où les gradients de vitesse sont maximaux, le schéma tiré du modèle de Cola (fig. 6 ) indique une puissance moyenne dissipée par unité de volume de l'ordre de :

$$
\mathscr{P} \sim \frac{\rho q V_{0}^{2}}{\alpha d^{2}}=\frac{2 \rho g}{\alpha} \cdot \frac{q \cdot H}{d^{2}}
$$

et donc la profondeur $d$ est liée à cette puissance par

$$
\left[d \sim \sqrt{\frac{2 \rho g}{\alpha \cdot P}} \cdot q^{1 / 2} \cdot H^{1 / 2}\right]
$$

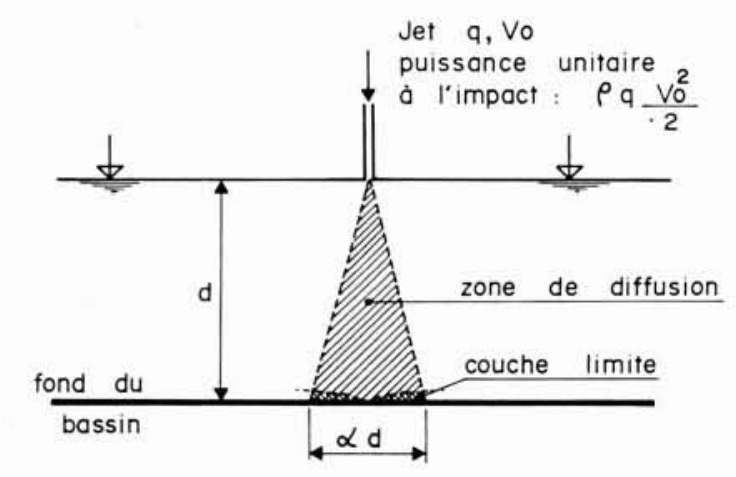

Figure 6 - Schéma de dissipation associé au modèle de CoLA. 
- Si l'on considère maintenant la dissipation concentrée au voisinage de la couche limite qui se développe à la base de la zone de diffusion (fig. 6), la puissance moyenne dissipée par unité de surface du fond dans ce voisinage est de l'ordre de :

$$
P^{\prime} \sim \frac{\rho q V o^{2}}{2 \alpha d}=\frac{\rho g}{\alpha \cdot} \cdot \frac{q \cdot H}{d}
$$

et donc la profondeur $d$ est liée à cette puissance par :

$$
d \sim \frac{\rho g}{\alpha \cdot J^{\prime}} \cdot q \cdot H
$$

- dans les expressions (12) et (14), le débit unitaire $q$ et la hauteur de chute $H$ ont le même poids, or il a été noté en introduction un trait commun à nombre d'expressions empiriques de la profondeur limite : le poids du débit unitaire est le plus fort.

Si la profondeur ultime est effectivement décrite par une des formules empiriques mentionnées en introduction, les remarques précédentes suggèrent donc que la dissipation d'énergie ne serait pas vraiment le moteur du mécanisme.

Cependant, il se peut que la distribution des niveaux relatifs de fluctuation de pression - les fonctions $\lambda^{+}$et $\lambda^{-}$ - dépende de la puissance unitaire dissipée par turbulence au voisinage du fond; les résultats publiés par FRANZETTI couvrent un éventail de situations peut être un peu étroit pour permettre l'évaluation d'un tel effet.

Enfin, les effets d'échelle ont une influence certaine sur le débit relatif de l'air entraîné naturellement dans le bassin par le jet (ERvine (1976) [13]). De ce fait, on peut penser que les effets d'échelle ont également une influence sur la dissipation, facilitée par l'entrainement d'air (BıN. DER et al. (1980) [12]), ainsi que sur la propagation et la distribution en intensité des fluctuations de pression, certainement sensibles à la compressibilité de l'eau émulsionnée d'air.

\section{Conclusion}

- Le schéma physique proposé dans cet article fait donc apparaître que :

- le moteur du mécanisme de formation des fosses d'érosion est l'effet de quantité de mouvement, c'est-à-dire les efforts de pression dûs à la déflexion des lignes de courant;

- la dissipation d'énergie intervient comme moyen intermédiaire, par les fluctuations de pression associées à la turbulence entretenue par cette déflexion du jet;

- le moyen d'action est la cavitation produite par les fluctuations en dépression.

La cavitation attaquant les matériaux les plus tenaces, la profondeur ultime donnée par ce mécanisme est, au moins, la profondeur limite de cavitation, indépendante de la nature du matériau formant le fond.

- La formation d'une fosse étant le résultat de l'intéraction entre un écoulement particulier avec un fond donné, il est clair que d'autres effets interviennent, tels que l'effet abrasif des éléments entraînés, et que la nature du fond a une influence certaine sur le résultat.

Cependant, le mécanisme physique lié au seul écoulement d'eau qui est proposé ici, malgré son simplisme, donne un ordre de grandeur vraisemblable; l'objectif de cet article était de montrer comment.

- Ce mécanisme ne suggère guère d'autres palliatifs pratiques que ceux qui sont pratiqués depuis longtemps :

- soutien du niveau aval par un contre barrage pour créer une épaisseur d'eau amortissant l'impact;

- étalement et fragmentation du jet afin de répartir l'impact et d'éviter la concentration d'efforts de poinçonnement:

- aération afin d'amortir les fluctuations de pression et de limiter les risques de cavitation tout en facilitant la dissipation d'énergie.

\section{Références}

[1] (Réf. cit. in [2]) "Erosioni di fondo a valle di uno scarico" A. VerONESE - Annali dei Lavore Publici. Roma - Settembre 1937, p. 77.

[2] E. SCIMEMI (1947) - Discussion in Transactions A.S.C.E. Vol. 112-1943, pp. 1016-1019.

[3] U.S. Bureau of Reclamations "Design of small dams". Ed. 1961 , p. 307

[4] R.B.F. MARTINS (1975) - "Scouring of rocky riverbeds by free-jet spillways" - Water Power \& Dam Construction, April 1975.

[5] L.I. MaChado (1982) - "O sistema de dissipassao de energia proposto para a barragem do Xingo" - C.R. Symposium international sur les barrages en vallée étroite - Rio de Janeiro - 1982, Vol. 1.

[6] H. Chandra, B.M. Agarwal (1982) - "Lay out of Lakhwar dam spillway" - C.R. Symposium international sur les barrages en vallée étroite - Rio de Janeiro - 1982, Vol. 1.

[7] P.J. MASON (1984) - "Erosion of plunge pools downstream of dams due to the action of free-trajectory jets" - Proc. Instn. Civ. Engrs Part 1 - 1984-76, May, pp. 523-537.

[8] A. LencAstre (1985) - "Etat des connaissances sur le , dimensionnement des évacuateurs de crue des barrages " La Houille Blanche, no 1-1985, p. 19-51.

[9] R. COLA (1966) "Diffusione di un getto piano verticale in un bacino d'acqua d'altezza limitada " - L'Energia Elettrica Vol. XLIII

[10] S. Franzetti (1980) « Pressioni idrodinamiche sul fondo di una vasca di smorzamento " - L'Energia Elettrica, No 6.

[11] H.L. Albertson, Y.B. Dal, R.A. Jensen, H. Rouse (1950) "Diffusion of submerged jets" - Transactions A.S.C.E. Paper 2409, Vol. 115.

[12] Binder, Duport, Meilland, Ramette, Valibouse (1980) "Problèmes généraux de la dissipation d'énergie dans les ouvrages et circuits hydrauliques " - La Houille Blanche n० 7/8-1980, p. $423-430$

[13] D.A. ERviNE (1976) - «The entrainment of air in water " - Water Power \& Dam Construction - December 1976 


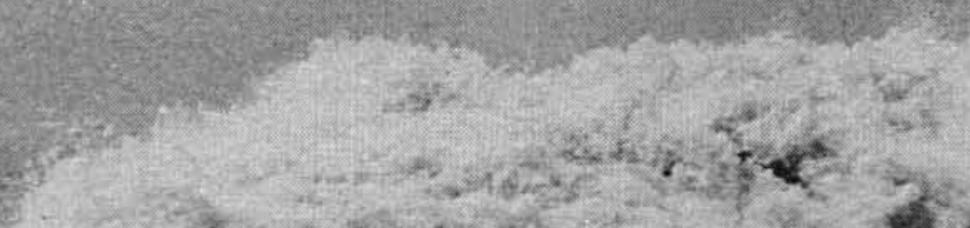
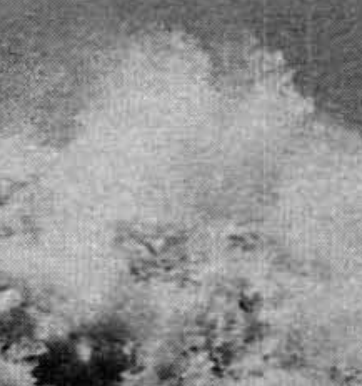

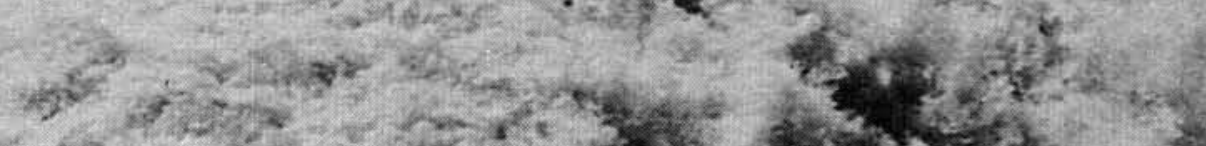

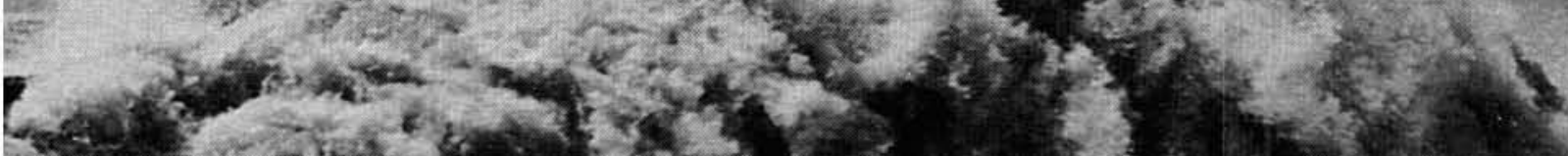
जace tere

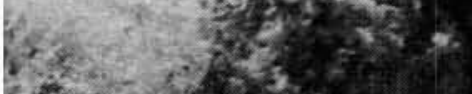

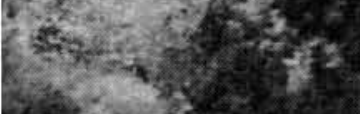

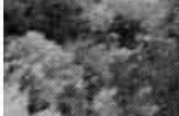

$3+x^{2}$

स्रत

s.

13.

3

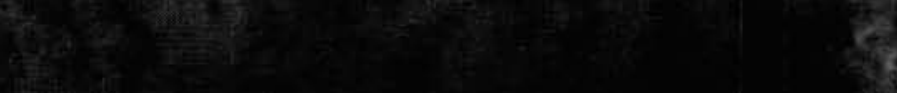
1.

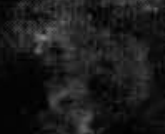
$x+2 x^{2}$
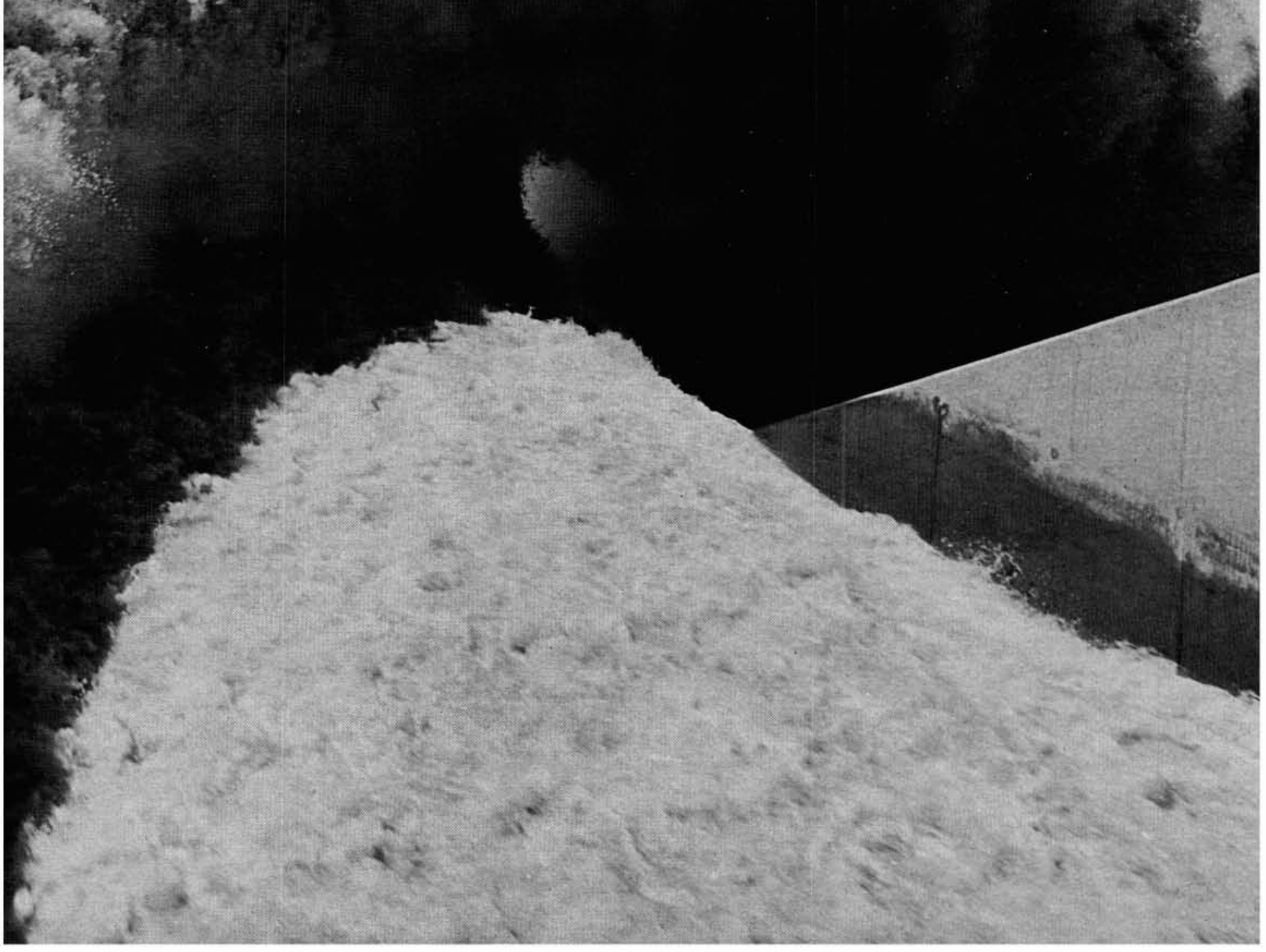